Care of the Child with Tympanostomy Tubes: A Guide for the Primary Care Physician PA Pitcher 1 , BSc., MD '99, MM Carr 2 , DDS, MD, MEd, KD Clarke 3, MD, FRCSC 1,2 Department of Otolaryngology, Dalhousie University, 3 Department of Otolaryngology, Dalhousie University; Izaak Walton Killam Children's Hospital, Halifax, Nova Scotia

\begin{abstract}
Tympanostomy tube (TT) placement in children for the treatment of chronic otitis media with effusion (COME) and recurrent acute otitis media (RAOM) is the most common surgical procedure performed among otolaryngologists. Despite its popular-ity, the literature is lacking in information for family doctors regarding many aspects of the procedure, including indications, complications, and post-operative patient care. In this review, we discuss COME, RAOM, rationale and indications for TT placement and complications of TT placement. With respect to the complications of TT placement there will be an emphasis on management of post-operative otorrhea, and post-operative patient care.
\end{abstract}

Myringotomy with insertion of tympanostomy or ventilation tubes (TT) as a potential component of the treatment plan for chronic otitis media with effusion (COME) and recurrent acute otitis media (RAOM) is the most common surgical procedure performed in children that requires general anaesthesia (see Figure 1) (1). Insertion of TT has been accepted since they were reintroduced by Armstrong in 1954 and has been gaining in popularity ever since (2). It has been reported that, $30.11 \%$ of children younger than 24 months underwent TT placement in an 8 month period (3), demonstrating that it is important for family doctors to know how to appropriately manage patients with TT placement during their post-operative course. Postoperative patient care remains an under represented topic in the literature. Therefore here we discuss otitis media infection, indications for tube surgery, post-operative patient care, and complications of tube surgery.

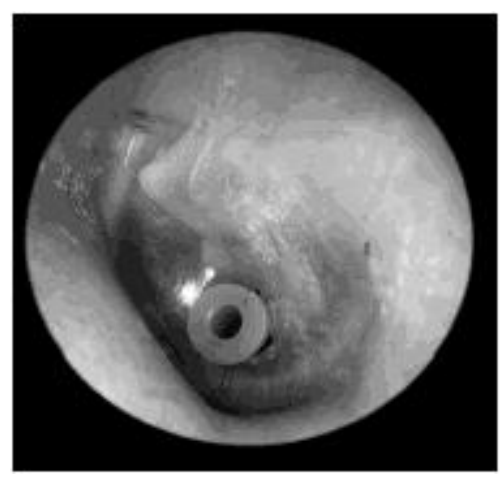

Figure 1. Left tympanic membrane with tympanostomy tube in situ. Photo courtesy of Dr. Michael Hawke MD 
FRCSC and used with permission.

\section{CHRONIC AND RECURRENT ACUTE OTITIS MEDIA}

Otitis media is one of the most common diseases of childhood. Epidemiologists in the United States at the Centres for Disease Control and Prevention (CDC) identified 24.5 million visits at which the principle diagnosis was otitis media (4). Diagnosis of otitis media in office practices more than doubled from 1975 to 1990 (4).

Acute otitis media (AOM) represents the rapid onset of an inflammatory process of the middle ear space (5). One or more local or systemic signs are present: otalgia (or pulling on the ear in the young infant), imbalance/falls (in toddlers), otorrhea, fever, recent onset of irritability, anorexia, vomiting or diarrhea (1). The tympanic membrane is full or bulging, opaque and has limited or no mobility with pneumatic otoscopy - indicative of a middle ear effusion. Erythema of the eardrum is an inconsistent finding. Following an episode of acute otitis media, a middle ear effusion that persists for longer than three months is termed chronic otitis media with effusion (1).

Chronic otitis media with effusion (COME) usually results in conductive hearing loss. The tympanic membrane may be thickened, opaque and show reduced mobility with pneumatic otoscopy. Air bubbles may be observed through the tympanic membrane (6).

A careful history and physical exam will lead to the diagnosis of otitis media. A careful history should elicit systemic signs of AOM. Less frequently otorrhea, vertigo, facial paralysis or swelling of the post-auricular area (mastoid) may occur as complications of AOM. In COME hearing loss may be the only symptom. A complete exam of the head and neck should be performed in every patient suspected to have otitis media. In patients with unilateral serous otitis media, the nasopharynx should be visualized to rule out the possibility of a tumour $(5,6)$. Otoscopy and tympanometry are part of the physical examination to establish the diagnosis of otitis media. The ultimate diagnostic test to confirm the presence of otitis media involves aspiration of middle ear contents for culture. Audiometry demonstrating conductive hearing loss contributes to confirmation of middle ear fluid $(5,6)$.

The pathogenesis of acute otitis media likely follows this sequence of events: the patient has an antecedent event (usually a viral upper respiratory tract infection) that results in congestion of respiratory mucosa throughout the respiratory tract, including nose, nasopharynx, eustachian tube and middle ear; congestion of the mucosa in the eustachian tube results in obstruction of the narrowest part of the tube, the isthmus. This obstruction results in negative pressure and then the development of a middle ear effusion, which has no exit and accumulates in the middle ear. The virus causing the primary upper respiratory tract infection and the potentially pathogenic bacteria that colonize the nasopharynx can enter the middle ear through the eustachian tube and cause an acute suppurative otitis media (1). 
Results from the Pittsburgh Otitis Media Study Group show Streptococcus pneumoniae and Haemophilus influenzae to be the most frequent bacterial pathogens, followed by Moraxella catarrhalis. Group A streptococcus, Staphylococcus aureus and gram negative enteric bacilli are infrequent causes of otitis media (7). In the summer months, children are more likely to get otitis externa, with Pseudomonas aeruginosa being the most frequently colonized bacteria (1).

\section{RATIONALE FOR THE USE OF TYMPANOSTOMY TUBES}

Results of several clinical trials have shown that TT can be beneficial in selected infants and children, since middle ear disease is reduced and hearing is restored, although there are known complications of the surgery (8-11). A functioning TT would maintain ambient pressure within the middle ear and mastoid and provide adequate drainage down the eustachian tube and, in AOM through the TT. Therefore drainage and ventilation function of the eustachian tube are improved by the TT (1). The TT also allows topical antibiotic treatment for AOM to reach the middle ear. The protective function of the eustachian tube may be impaired by TT placement because reflux of nasopharyngeal secretions into the middle ear may be enhanced when a TT eliminates the middle ear air cushion; this can result in reflux otitis media (1).

\section{INDICATIONS FOR TYMPANOSTOMY TUBES}

There remains uncertainty about the efficacy and safety of tube placement by many clinicians and investigators $(12,13)$. Results of clinical trials are now available to arrive at criteria for insertion of tubes for chronic otitis media with effusion (8-10).

Indications for TT placement have been published by the American Academy of Otolaryngology-Head and Neck Surgery (AAO-HNS) (14). The 1995 (most recently published) indications for TT placement from the AAO-HNS are shown in Table 1. The AAO-HNS emphasizes that these clinical indicators should not be deemed inclusive of all proper treatment decisions or methods of care, nor exclusive of other treatment decisions or methods of care reasonably directed to obtaining the same results. These clinical indicators serve as a check list for practitioners and a quality care review tool for clinical departments.

TT placement immediately corrects the conductive hearing loss associated with a middle ear inflammatory process and decreases the patient's tendency toward reinfection $(5,6)$. Patients who have bilateral otitis media with effusion, which has been unresponsive to antimicrobial agents for three months or greater are eligible for TT placement. For children with unilateral OME, the duration can be extended to six months since hearing is assumed to be good in the unaffected ear $(1,14)$

Many children have recurrent episodes of acute otitis media that respond to antimicrobial treatment. It would be beneficial to prevent these infections from occurring since they affect hearing when effusion is present and the child is uncomfortable with fever and otalgia. Three or more episodes in a 6 month period or at least four episodes during a period of 1 year are indications for placing TT (11). 
Tympanostomy tubes are also indicated in the following cases: 1. eustachian tube dysfunction resulting in one or more of the following: significant and symptomatic hearing loss $(1,14,15)$, otalgia, vertigo (16), tinnitus, or severe atelectasis, especially in those ears in which a retraction pocket is present in the posterosuperior quadrant or pars flaccida, or both (17); 2. following tympanoplasty, when eustachian tube function is known to be poor (14); and 3. when a complication of otitis media is present, such as facial paralysis, meningitis, coalescent mastoiditis or brain abscess $(1,14)$. An infrequent indication for TT placement is prevention of barotitis trauma for unconscious children who require hyperbaric treatment for smoke inhalation and/or carbon monoxide poisoning $(14,18)$.

Absolute contraindications to surgery have not been defined but it is agreed by most otologists that severe eustachian tube dysfunction lessens the possibility of hearing restoration in most cases (6).

\section{Table 1. Indications for Myringotomy and Tympanostomy Tubes from 1995 American Academy of Otolaryngology-Head and Neck Surgery Clinical Indications Compendium (14)}

\section{History}

a. Severe acute otitis media (myringotomy).

b. Hearing loss $>30 \mathrm{~dB}$ in patient with otitis media with effusion (myringotomy or tube).

c. Poor response (describe) to antibiotic for otitis media (myringotomy or tubes).

d. Impeding mastoiditis or intra-cranial complication due to otitis media (myringotomy).

e. Otitis media with effusion $>3$ months (myringotomy or tympanostomy tube).

f. Recurrent episodes of acute otitis media (more than 3 episodes in 6 months or more than 4 episodes in 12 months) (tympanostomy tube).

g. Chronic retraction of tympanic membrane or pars flaccida (tympanostomy tube).

h. Barotitis syndrome.

i. Autophony due to patulous eustachian tube.

\section{Physical Exam}

a. Description of tympanic membrane.

b. Evaluation of middle ear.

\section{Tests}

a. Audiometry - pure tones or speech reception threshold.

b. Tympanometry. 
c. Mastoid imaging (in case of suspected mastoiditis).

A medical evaluation prior to ear surgery begins with a complete nose and throat evaluation. The medical history may suggest the presence of otosclerosis, dietary deficiencies, poor environment conditions or presence of genetic or constitutional predisposition to upper respiratory disease, all of which may have an affect on the surgical outcome and recovery. Hypothyroidism, hypoestrogenism, vitamin deficiency and serum protein deficiency may all influence the operative result. Bleeding problems endanger success of the operation. Culture of secretions from the middle ear can be performed. Radiological exam is of value in revealing hidden areas of mastoid disease or anatomic variations influencing the course of surgery, but this is not routine or considered necessary prior to TT insertion. Otoscopy, tympanometry and audiometry are typically performed preoperatively $(5,6)$.

\section{COMPLICATIONS}

Placement of TT usually requires children to receive a general anaesthetic. There are known potential complications of general anaesthetics, such as allergic reaction and respiratory depression. In a study evaluating possible complications of general anaesthetic in 510 children who had tubes placed, no complications were reported in $83 \%$ (19). In 12\% there was a minor degree of airway obstruction during the procedure; no serious complications or deaths were reported. Thus, the surgery itself is considered relatively safe.

Table 2. Early and Late Complications of Tympanostomy Tube Placement

\section{Early Complications:}

Persistent otorrhea $(1,20-24,37,38)$

Blockage of the tube $(34,35)$

Early extrusion

Hearing loss $(1,13)$

Ossicular disruption (37)

\section{Late Complications}

Persistent perforation after tube extrusion $(9-11,37,38,41,42)$

Scarring of the tympanic membrane $(1,37,38)$

Atrophic monomeric TM $(1,37,38)$

Granuloma $(37,38)$

Tympanosclerosis $(1,37)$

Cholesteatoma $(1,37,38)$

Migration of tympanostomy tube into middle ear canal (37) 
Complications of TT may be divided into two groups. The first group includes early complications, which occur while the tube is in place and patent and appear to be directly related to the tube. The second group includes late complications that occur after the tube has extruded and may or may not be related to the tube (Table 2).

\section{OTORRHEA}

The most common complication of TT placement is post-operative tube otorrhea, which occurs in 6 to 40 percent of cases (20-22). This may occur in up to 10\% of children within two weeks of surgery (23). Delayed otorrhea has been reported in $25 \%$ of children with tubes placed for chronic effusion (24). A recent metanalysis reported that use of ototopical drops at the time of surgery was effective in reducing the rate of tube otorrhea, especially when the middle ear contained mucoid or purulent effusion (25). Phyicians should be cautioned in their use of these antibiotic drops because they are potentially ototoxic. Despite this concern, there is no evidence in the literature that short-term use is related to ototoxicity, therefore most otolaryngologists commonly prescribe these agents on a short-term basis for otorrhea (Table 3)(26).

\section{Table 3. Management Of Post-Operative Otorrhea at the Department of Otolaryngology,} Izaak Walton Killam Chil-dren's Hospital

\section{Acute}

Garasone TM (3-4 drops in affected ear for 5-10 days after surgery)

\section{Chronic}

Investigations:

CBC

Cultures of canal and middle ear

Antibodies to tetanus and pneumococcus

Immunoglobulin levels: IgG, A, M, E \& IgG subclasses

Treatment:

TimentinR (50 mg/kg every 6 hours) (28)

ClavulinR (50 mg/kg/day) (28)

Post-operative otorrhea is usually the result of reflux of nasopharyngeal secretions into the middle ear or from contaminants from the external canal, and not related to the operative procedure $(1,23)$. When otorrhea occurs, the fluid should be cultured from the middle ear by obtaining an aspirate through the tympanic membrane. A culture and cleaning of the ear canal should precede the aspiration of the middle ear. An immunodeficiency work up should also be considered in these patients because studies have shown that children with immunoglobulin 
deficiencies commonly develop recurrent acute otitis media. These children are less responsive to treatment with tympanostomy tubes (27). Investigations involved in managing chronic postoperative otorrhea in children who have TT in place are shown in Table 3.

Many practitioners believe that both oral and topical antibiotics should be initiated for chronic otorrhea. The usual initial treatment for AOM has been amoxicillin, $40 \mathrm{mg} / \mathrm{kg} / 24$ hour in three divided doses or oral ampicillin, 50-100 mg/kg/24 hours in four divided doses for 10 days. In children allergic to penicillin, a combination of erythromycin, $40 \mathrm{mg} / \mathrm{kg} / 24$ hours and sulfisoxazole, $120 \mathrm{mg} / \mathrm{kg} / 24$ hours in four divided doses may be substituted. If betalactamase

producing $\mathrm{H}$. influenza or B. catarrhalis is suspected then either amoxicillin-clavulanate 40 $\mathrm{mg} / \mathrm{kg} / 24$ hours in three divided doses, or trimethoprim-sulfamethoxazole, $8 \mathrm{mg}$ trimethoprim and $40 \mathrm{mg}$ of sulfamethoxazole every 24 hours may be used in two divided doses. Cefixime (Suprax, Lederle Labs., Wayne, NJ) $8 \mathrm{mg} / \mathrm{kg}$ in one dose or cefprozil (Cefzil, Bristol-MyerSquibb, Prinston, NJ) $15 \mathrm{mg} / \mathrm{kg} / 24$ hours may also be used effectively $(5,6)$. At the Izaak Walton Killam (IWK) hospital, Garasone TM, 3-4 drops are placed in the affected ear for 2 to 3 days after surgery, 4 times a day to prevent post-operative otorrhea (28) (Table 3). If children have persistent otorrhea at a 2 week post-operative check, Timentin R, $50 \mathrm{mg} / \mathrm{kg}$ every 6 hours or Clavulin R, 50 mg/kg/day are prescribed (28) (Table 3).

With recurrent episodes of AOM in the presence of a functioning TT, antimicrobial prophylaxis should be given to prevent recurrent middle ear infections and otorrhea $(29,30)$. When otorrhea fails to respond to medical management, granulation tissue around the tube, an occult cholesteatoma or unusual pathogens such as Candida albicans (31), Actinomyces (32) or Aspergillus (33) should be suspected.

\section{EARLY/DELAYED EXTRUSION}

Most tubes remain in the tympanic membrane for 6 to 12 months, although some have been known to remain in place for years (9-11). Early extrusion of TT is a potential complication and results in repeated tube surgery and exposure to anaesthetic and its associated risks.

\section{TUBE BLOCKAGE}

Tubes can be blocked immediately after surgery by blood or thick middle ear secretions $(34,35)$. Results of a prospective study found that antibiotic ointment application to the TT at the time of surgery did not significantly reduce the rate of tube blockage with blood (36).

\section{TYMPANIC MEMBRANE CHANGES}

Scarring of the tympanic membrane, tympanosclerosis and localized or diffuse membrane atrophy, with or without retraction pockets, or atelectasis may occur following TT placement $(37,38)$. Results of a study comparing anatomic and audiologic sequelae after repeated TT insertion or prolonged antibiotic therapy for otitis media, showed that tympanosclerosis (52.3\%), tympanic atrophy (40.7\%) and hearing threshold elevation greater than $20 \mathrm{~dB}$ (9.3 to 18.7\%) 
occurred at significantly greater rates than in their medically treated comparison group (13). Tympanic calcifications and severe retraction were reported as late complications of TT insertion (39). Tympanosclerosis could theoretically impair the transmission of higher frequencies and shift the resonance point toward low tones, however this has not been reported to have a clinical effect on hearing test results (37).

The incidence of retraction of tympanic membrane is low. In one study, retraction of the tympanic membrane following extrusion of the TT was reported in about $1.6 \%$ of TT insertions (38). This study also discussed that retraction might not be called a complication because it causes no symptoms, but it was demonstrated in another study that retraction pockets may progress to cholesteatomas $(38,40)$.

\section{TYMPANIC MEMBRANE PERFORATION}

Following extrusion of a tube, a perforation site may remain at the insertion site. This is less common than tympanosclerosis, with the rate of perforation ranging from 0.5 to 25 percent depending on the type of tube and the number of tubes inserted into the same tympanic membrane over time. Long term tubes have the highest rate of tympanic membrane perforation and short term tubes have a lower frequency $(41,42)$. No preoperative factor completely predicts the development of perforation (43). Clinical trials evaluated the safety and efficacy of tube insertion to treat or prevent chronic otitis media in 215 infants and children. Following spontaneous extrusion 14.8 percent of perforations at the tube site eventually closed while 1.4 percent of children required tympanoplasty after the perforation failed to close after two years (911).

\section{CHOLESEATOMA}

There are reports that a cholesteatoma may develop, usually at the site of tube insertion, either by invagination of squamous epithelium, or from a retraction pocket that develops due to persistent eustachian tube dysfunction $(1,37,38)$. Other studies showed no development of a cholesteatoma (39).

Table 4. American Academy of Otolaryngology-Head and Neck Surgery Tympanostomy Tube Placement Preoperative Patient Information (14)

Myringotomy with or without tympanostomy tube insertion is the most commonly performed ear operation. It is ex-tremely safe and effective.

Complications are minor and usually in the form of infec-tion, which may be treated with antibiotics. The tube usu-ally remains in place for several months, although it may be rejected sooner or remain in place for years. As long as there is a tube in the ear, care should be taken to avoid water contamination, especially bath water, by the use of ear plugs or, if desired, a bathing cap. Occasionally the ear drum fails to heal after tubes have been removed, and the resulting per- 
foration may require surgical repair.

In some cases, particularly when there is a family history of chronic ear disease, tympanostomy tubes may need to be re-placed.

Hearing improvement is usually immediate after fluid has been removed from the ear. Failure to improve hearing indi-cates a second problem in the middle or inner ear.

\section{OTHER COMPLICATIONS}

Granulation tissue formation and polyps have been reported as complications of TT insertion. Bleeding from the ear canal is usually the sign that alerts the parent to the problem. No sequelae results from a granuloma that resolves with removal or extrusion of the tube (37). Ossicular chain disruption is a possible problem, and sensorineural hearing loss (SNHL) could also occur (37), but these complications are extremely rare. Migration of the TT into the middle ear canal could occur, however, this is also rare. In one retro-spective study, one case out of 838 total myringotomy and tube insertions migrated into the tympanic cavity (37).

Fortunately, most of the potential complications to TT placement surgery are rare and not lifethreatening, which relieves anxiety parents may have prior to their child's surgery. Parents of children who will be undergoing TT place-ment surgery can be given information about the complica-tions of the surgery (Table 4).

\section{POSTOPERATIVE PATIENT CARE TUBE APPEARANCE AND FUNCTION}

Patients who had TT placement are reexamined approximately 2 weeks after insertion, at which time another audiogram is obtained and compared with the preoperative test. If hearing is normal and the TT are in place and patent (Figure 1), the child can be reexamined in 3 to 6 months and then every 3 to 4 months until spontaneous tube extrusion (44).

There are two general types of tubes: short-term tubes (intended to remain in the eardrum for 815 months) and long-term tubes (intended to remain in the eardrum >15 months) (Figure 2). In order to maintain position in the eardrum, tubes have inner and outer flanges (grommet tubes) or shafts too long to fit in the middle ear (T-tubes). Long-term tubes have large inner flanges, and shorter outer flanges to accumulate keratin and be extruded, or both. Tubes can be placed in the pars tensa of the tympanic membrane in any location except the posterior superior quadrant which overlies the incus and stapes (45).

A tympanostomy tube can function properly if it spans the eardrum, the tube lumen is patent, and no middle ear effusion is present. Tube function can be assessed through otoscopic examination which includes pneumatic otoscopy or tympanometry. On pneumatic otoscopy the normal eardrum should be mobile, if the eardrum with a tube in place is not mobile with no other signs of middle ear effusion, the tube is probably functioning properly. A flat tympanogram with large volume indicates that the tube is patent or there is a perforation elsewhere in the tympanic 
membrane. A peaked tympanogram indicates that the tube is clogged or extruded with a normal ear cavity (no effusion in the ear cavity). A flat tympanogram with a small volume indicates a nonfunctioning tube with a middle ear effusion (45).

\section{WATER PRECAUTIONS}

The issue of water precautions to be taken in children with tubes in place remains a widely debated topic among otolaryngologists (46). Results from a recent survey of otolaryngologists regarding their recommendations of water precautions in children with tubes showed that $13.1 \%$ forbid children with tubes to swim, $3.1 \%$ felt that no water precautions were necessary, and $68 \%$ recommended no deep diving or swimming below $180 \mathrm{~cm}$. Fifty-three percent of the survey respondents recommended earplugs as protection, with $67 \%$ recommending custom fit ear molds (46). Prophylactically applied ear drops with antimicrobials have also been suggested as a form of water protection.

Many studies and reviews have been published with attempts to evaluate the relationship between water exposure (in the form of swimming or bathing) and recurrent otorrhea in children with tubes (46-50). A recently published five-year prospective controlled study concluded that children with tubes who surface swim and do not dive receive no additional benefit from taking of water precautions in the form of earplugs or antibiotic drops (46). There were numerous flaws with this study in terms of the population studied and study design so there remains the need for further research in this area (51). To date, no universal water precaution guidelines exist in the literature and no study has shown that swimming with tubes increases the risk of otorrhea.

Based on the available published work, Table 5 shows water precautions for children with tympanostomy tubes.

\section{Table 5. Recommended Water Precautions* in Children with Tympanostomy Tubes in Place Derived From Current Literature}

1. Surface swimming (not swimming below $180 \mathrm{~cm}$ depth) in fresh water and chlorinated pools is allowed without earplug protection. If swimming more than $180 \mathrm{~cm}$ below the surface or deep diving children should wear earplugs (46-49).

2. Children should wear earplugs when washing hair and there should be no head submergence in soapy bath water without earplug protection (46-48).

3. If soapy water or shampoo is allowed to enter into the ear canal antibiotic drops should be applied (46).

*Earplug protection includes: 1) custom made or premanufactured earplugs or 2) earplugs plus a bathing cap. Silly Putty for water protection in children with tubes is strongly discouraged (52). 


\section{CONCLUSIONS}

1. Tympanostomy tube insertion is a common procedure.

2. The most common indications for tympanostomy tube surgeries are RAOM (three or more episodes of OM in a 6 month period or 4 episodes during a 1 year period) and COME (bilateral OME which has been unresponsive to nonsurgical therapy for three months or greater), with an associ-ated hearing loss.

3. Post-operative otorrhea is the most common com-plication from tympanostomy surgery and is treatable.

4. Patients are examined at 2 weeks post-operatively at which time an audiogram is obtained. If hearing is normal the child is re-examined in 3 to 6 months. Pneumatic otoscopy and tympanography are performed to assess tube function.

5. Water precautions in children with tympanostomy tubes in place include: no earplugs needed if surface swimming and no deep diving; earplugs needed when ears are immersed in soapy water or shampoo; antibiotic drops applied if soapy water or shampoo enters the ear canal.

\section{REFERENCES}

1. Bluestone CD, Klein JD.Otitis Media in Infants and Children 2nd Ed. W.B. Saunders Co., Philadelphia, 1995.

2. Armstrong BW. A new treatment for chronic secretory otitis media. Arch Otolaryngol 1954; 59: 653-54.

3. Myer CM, France A. Ventilation tube placement in a managed care population. Arch Otolaryngol Head Neck Surg 1997; 123(2): 226-28.

4. Schappert SM. Office visits for otitis media: United States, 1975-90. From Vital and Health Statistics of the Centers for Disease Control/ National Center for Health Statistics 1992; 214: 118.

5. Healy GB. Otitis media and middle ear effusions. In Ballenger JJ, Snow JB (Eds.) Otorhinolaryngology: Head and Neck Surgery 15th Ed. Williams and Wilkins, Baltimore 1996; pp1003-9.

6. Austin DF. Chronic otitis media. In Ballenger JJ, Snow JB (Eds.) Otorhinolaryngology: Head and Neck Surgery 15th Ed. Williams and Wilkins, Baltimore 1996; pp1010-19.

7. Bluestone CD, Stephenson JS, Martin LM. Ten year review of otitis media pathogens. Pediatr infect Dis J 1992; 11(8 suppl): s7-s11.

8. Gates GA, Avery CA, Prihoda TJ, Cooper JC Jr. Effectiveness of adenoidectomy and tympanostomy tubes in treatment of chronic ototis media with effusion. N E J M 1987; 317(23): 1444-51.

9. Mandel EM, Rochette HE, Bluestone CD, Paradise JL, Nozza RJ. Myringotomy with and without tympanostomy tubes for chronic otitis media with effusion. Arch Otolaryngol Head Neck Surg 1989; 115: 1217-24.

10. Mandel EM, Rochette HE, Bluestone CD, Paradise JL, Nozza RJ. Efficacy of myringotomy with and without tympanostomy tubes for chronic otitis media with effusion. Pediatr Infect Dis J 1992; 11: 270-77. 
11. Casselbrant ML, Kaleida PH, Rochette HE, Paradise JL, Bluestone CD, Kurs-Lasky M, et al. Efficacy of antimicrobial prophylaxis and of tympanostomy tube insertion for prevention of recurrent acute otitis media: Results of a randomized clinical trial. Pediatr Infect Dis J 1992; 11(4): 278-86.

12. Bodner EE, Browning GG, Chalmers FT, Chalmers TC. Can meta-analysis help uncertainty in surgery for otitis media in children? J Laryngol Otol 1991; 105(10): 812-819.

13. Pichichero ME, Berghash CR, Hengerel AS. Anatomic and audiologic sequelae after tympanostomy tube insertion or prolonged antibiotic therapy for otitis media. Pediatr Infect Dis J 1989; 8(11): 780-87.

14. Clinical Indicators for Otolaryngic- Head and Neck Surgery. American Academy of Otolaryngology-Head and Neck Surgery, Inc., 1995.

15. Cotton RT. The surgical management of chronic otitis media with effusion. Pediatric Ann 1991; 20(11): 628-37.

16. Jones NS, Prichard AN, Radomskij P, Snashall SE. Imbalance and chronic secretory otitis media in children: Effect of myringotomy and insertion of ventilation tubes on body sway. Ann Otol Rhinol Laryngol 1990; 99(6 pt 1): 477-81.

17. Chen DA \& Luxford WM. Myringotomy and tube insertionfor relief of patulous eustachian tube symptoms. Am J Otol 1990; 11(4): 272-73.

18. Fernau JS, Hirsh BE, Derkay C, Ramasastry S, Schaefer SE. Hyperbaric oxygen therapy: effect on middle ear and eustachian tube function. Laryngoscope 1992; 102(11):48-52.

19. Markowitz-Spence C, Brodsky L, Syed N, Stanievich J, Volk M. Anesthetic complications of tympanostomy tube placement in children. Arch Otolaryngol Head Neck Surg 1990; 116(7):

809-12.

20. Balkany TJ, Barkin RM, Suzuki BH, Watson WJ. A prospective study of infection following tympanostomy and tube insertion. Am J Otol 1983; 4(4): 288-91.

21. Klingensmith MR, Strauss M, Conner GH. A comparison of retention and complication rates of large-bore (Paparella II) and small-bore middle ear ventilation tubes. Otolaryngol Head Neck Surg 1985; 93(3): 322-30.

22. Slack RW, Gardner JM, Chatfield C. Otorrhea in children with middle ear ventilating tubes: a comparison of different types of tubes. Clin Otolaryngol 1987; 12(5):357-60.

23. Scott BA, Strunk CL. Posttympanostomy otorrhea: the efficacy of canal preparations. Laryngoscope 1992; 102(10): 1103-7.

24. Gates GA, Avery CA, Cooper JC, Prihoda TJ. Chronic secretory otitis media: effects of surgical management. Ann Otol Rhinol Laryngol Suppl 1989; 98 (suppl 138):2-32.

25. Garcia P, Gates GA, Schechtman KD. Does topical antibiotic prophylaxis reduce posttympanostomy tube otorrhea? A meta-analysis. Ann Otol Rhinol Laryngol 1994; 103(1): 54-8.

26. Lundy LB \& Graham MD. Ototoxicity and ototopical medications: A survey of otolaryngologists. Am J Otol 1993; 14(2): 141-46.

27. Masin JS, Hostoffer RW, Arnold JE. Otitis media following tympanostomy tube placement in children with IgG2 deficiency. Laryngoscope 1995, 105(11):1188-90 
28. Compendium of the Pharmaceuticals and Specialties 32nd Ed. Canadian Pharmaceutical Association 1997. 29. Hester TO, Jones RO, Archer SM, Haydon RC. Prophylactic antibiotic drops after tympanostomy placement. Arch Otolaryngol Head Neck Surg 1995; 121(4): 445-48. 30. Ramadan HH, Tarazi T, Zaytonn GM. Use of prophylactic otic drops after tympanostomy insertion. Arch Otolaryngol Head Neck Surg 1991; 117(5): 537.

31. Cohen SR, Thompson JW. Otitic Candidiasis in children: an evaluation of the problem and the effectiveness of ketoconazole in 10 patients. Ann Otol Rhinol Laryngol 1990; 99(6 pt 1): 427-31.

32. Shelton C, Brackmann DE. Actinomycosis otitis media. Arch Otolaryngol Head Neck Surg 1988; 114(1): 88-9.

33. Hall PJ, Farrior JB. Aspergillus mastoiditis. Otolaryngol Head Neck Surg 1993;108(2): 16770 .

34. Gibb AG. Long-term assessment of ventilation tubes. J Laryngol Otol. 1980; 94(1): 39-51.

35. Jamal TS. Avoidance of postoperative blockage of ventilation tubes. Laryngoscope 1995; 105(8 pt 1): 833-4.

36. Cunningham MJ, Harley EH. Preventing perioperative obstruction of tympanostomy tubes: a prospective trial of a simple method. Int. J. Pediatr. Otorhinolaryngol 1991; 21(1):15-20. 37. Hughes LA, Warder FR, Hudson WR. Complications of tympanostomy tubes. Arch Otolaryngol 1974; 100(2):151-54.

38. McLelland CA. Incidence of complications from use of tympanostomy tubes. Arch Otolaryngol 1980; 106(2): 97-9.

39. Fior R, Veljak C. Late results and complications of tympanostomy tube insertion for prophylaxis of recurrent purulent otitis media in pediatric age. Int J Pediatr Otorhinolaryngol 1984; 8(2):139-46.

40. Buckingham RA, Ferrer JL. Reversibility of chronic adhesive otitis media with polyethylene tube, middle ear air-vent, kodachrome time lapse study. Laryngoscope 1966; 76(6):933-1014.

41. Todd GB. Audit of the incidence of persistent perforation of the tympanic membrane following grommet re-moval or extrusion. J Laryngol Otol 1993; 107: 593-596.

42. Bulkley WJ, Bowes AK, Marlowe JF. Complications following ventilation of the middle ear using Goode T tubes. Arch Otolaryngol-Head and Neck Surg 1991, 117(8):895-8.

43. Levine S, Daly K, Giebink GS. Tympanic membrane perforations and tympanostomy tubes. Ann Otol Rhinol Laryngol 1994; 163:27-30.

44. Bluestone CD, Stool SE. Otologic surgical procedures in: Atlas of Pediatric Otolaryngology . W.B. Saunders Co., 1995; pp28-128.

45. Isaacson G \& Rosenfeld RM. Care of the child with tympanostomy tubes: A visual guide for the pediatrician. Pediatrics 1994; 93(6 pt 1): 924-9.

46. Derkay CS, Shroyer MN, Ashby J. Water precautions in children with tympanostomy tubes. Am J of Otolaryngol 1992; 13(5): 301-5.

47. Salata JA \& Derkay CS. Water precautions in children with tympanostomy tubes. Arch Otolaryngol Head Neck Surg 1996; 122(3): 276-80. 
48. Pringle MB. Grommets, swimming and otorrhea - a review. J of Laryngol and Otolol 1993; 107(3): 190-94.

49. Parker GS, Tami TA, Maddox MR, Wilson JF. The effect of water exposure after tympanostomy tube inser-tion. Am J of Otolaryngol 1994; 15(3): 193-96.

50. Cohen HA, Kauschansky A, Ashkenasi A, Bahir A, Frydman MD, Horev MD. Swimming and grommets. J Fam Pract 1994; 38(1): 30-32.

51. Adelman A. Water precautions in children with tympanostomy tubes. J Fam Pract 1996; 42(6): 567-68.

52. Muntz HR. The use of silly putty as an ear plug. Arch Otolaryngol Head Neck Surg 1995; 121(3): 354. 